\title{
Poli-RRT*: optimal RRT-based planning for constrained and feedback linearisable vehicle dynamics
}

\author{
Matteo Ragaglia, Maria Prandini and Luca Bascetta
}

\begin{abstract}
This paper proposes a Rapidly exploring Random Trees planning strategy (Poli-RRT*) that computes optimal trajectories in presence of vehicle constraints (e.g., differential and actuation constraints) without approximating the nonlinear dynamics, but relying on exact linearisation. In this way, the optimal control problem that is introduced to determine the trajectories extending the tree can be expressed as a quadratic program and efficiently solved. Poli-RRT* is formulated and tested via simulation on a unicycle-like model of a vehicle subject to actuation constraints. Notably, the approach can be applied to any other feedback linearisable vehicle model, subject to different types of constraints.
\end{abstract}

\section{INTRODUCTION}

In the past few years autonomous aerial, underwater, and ground vehicles have gained considerable popularity in the robotics field, as they allow to perform critical tasks without endangering the life of humans. Their applications range from scientific exploration to provision of commercial services, from search and rescue to military operations, like for instance reconnaissance or intelligence gathering.

As application scenarios become more and more complex, the possibility to let human operators focus on high-level tasks, rather than on control of the vehicle, becomes more and more important. As a consequence, there is a strong perceived need for autonomy, in order to improve the whole system's efficiency, reliability, and safety.

Among the huge number of functionalities that are required to develop an autonomous vehicle, three are particularly important, i.e. localisation, planning, including obstacle avoidance, and trajectory tracking. Though they are all crucial to let the vehicle complete a task, the planner has the responsibility to compute a trajectory that takes the vehicle to the desired location, being also "feasible", i.e., compatible with the vehicle kino-dynamic constraints, and "safe".

Considering the state-of-the-art in planning techniques (see Section II for further details), sampling-based algorithms represent the most widespread "practical approach" to the planning problem. The outstanding success they achieved in the last decade is due to a rather simple yet effective idea: using a collision-checking module to provide information about feasibility of candidate trajectories. In this way, a set of points sampled from the free-space is connected in order to build a graph (roadmap) of feasible trajectories that are used to construct the solution to the original planning problem.

This work is partly supported by the European Commission under project UnCoVerCPS (grant number 643921). The authors are with Politecnico di Milano, Dipartimento di Elettronica Informazione e Bioingegneria, Piazza L. da Vinci, 32 - 20133 Milano, Italy \{matteo.ragaglia, maria.prandini, luca.bascetta\}@polimi.it
In most practical applications, however, one may be interested in finding paths of minimum cost, and in accounting for vehicle constraints like, for example, actuator limitations. Unfortunately, moving from a standard planning problem to an optimal planning problem or even to an optimal planning problem with constraints, computational intractability comes at no surprise. As a matter of fact, there have been several attempts [1]-[5] to propose an algorithm to solve an optimal planning problem with vehicle constraints, and all these approaches were based either on the shooting method [6] or on linearisation of the vehicle model.

The aim of this work is to propose Poli-RRT*, which is the first RRT-based planner that takes into account vehicle constraints but it does not need to represent vehicle dynamics with an approximate linearised model. In fact, the proposed methodology relies on an exact linearisation of the model that allows to efficiently recast the optimal control problem used to calculate the trajectories for extending the tree as a quadratic program, without any model simplification.

Poli-RRT* is formulated considering a unicycle-like vehicle and actuation constraints, but can be easily applied to any other feedback linearisable vehicle model with constraints of different type.

\section{RELATED WORK}

Two main approaches to address the planning problem have been developed in the literature: search-based and sampling-based techniques.

Search-based algorithms [7], [8] look for the sequence of actions that allow the vehicle to go from a start to a goal state in an optimal way, constructing a graph of states and searching a solution into the graph.

Sampling-based algorithms exploits the idea of sampling a continuous state space, instead of considering a pre-defined finite set of configurations, as it often occurs with searchbased methods. They proved to be very effective for planning in high-dimensional spaces since, even though they are not complete, they provide probabilistic completeness.

The most important sampling-based planners to date are Probabilistic RoadMaps (PRM) [9], [10] and Rapidlyexploring Random Trees (RRT) [11].

PRM algorithm is a multiple-query method that first constructs a graph (the roadmap) representing a set of collisionfree trajectories, and then answers queries by computing the shortest path connecting the initial state with the final state through the roadmap. PRM algorithms are probabilistically complete. 
Multiple-query methods, however, are valuable in a static, apriori known, highly structured environment, while in most on-line planning problems it is computationally challenging (or even unfeasible) to compute a roadmap a priori. This is the main reason behind the success of single-query counterpart to PRM: RRT.

First introduced in [11], RRT is an incremental samplingbased planning algorithm developed for searching highdimensional continuous state spaces. The incremental nature of RRT does not require to set the number of samples a priori, and returns a solution as soon as a path from the start to the goal state is found, enabling fast on-line implementations. Rapidly-exploring Random Graphs (RRG), RRT* and Optimal RRTs have been introduced in [1], [12]-[16] to address the optimal path planning problem, as well as path planning problems with complex task specifications. RRG algorithm builds incrementally a connected roadmap, randomly sampling the state space. Starting from RRG, RRT* can be obtained by simply removing cycles from graph, ensuring that vertices are reached through minimal-cost paths.

Not only RRTs represent an effective solution for the planning problem in case of linear robot dynamics, but they have been shown to work effectively for systems with differential constraints and non-linear dynamics [17]-[19]. In [1] the authors derive sufficient conditions to ensure asymptotic optimality of the RRT* algorithm for systems with differential constraints and show how to apply RRT* to a unicyclebased vehicle and to an holonomic double integrator robot. The same approach is generalised to arbitrary kino-dynamic systems in [2] by using the shooting method to connect pairs of states, thus obtaining feasible yet inherently suboptimal trajectories.

Other attempts to obtain a more sophisticated merge between RRT-based planning and optimality, in the case of non-linear dynamics, have also been recently proposed. At first [20] considers the problem of planning in continuous state and action spaces with non-linear deterministic dynamics.

In [3] the authors present an algorithm, named "Kinodynamic RRT*", that guarantees asymptotic optimality for any system characterised by linear differential constraints, in state spaces of any dimension. The same approach can be applied to non-linear dynamics as well, by using their first-order Taylor approximations.

Furthermore, in [4] the "LQR-RRT*" algorithm is proposed to solve planning problems with complicated or underactuated dynamics, by locally linearising the system and applying linear quadratic regulation (LQR).

Finally, in [5] a new method for applying RRT* to kinodynamic motion planning problems is introduced, using finite-horizon linear quadratic regulation (LQR) to measure cost and to extend the tree.

\section{PROPOSED APPROACH}

In this section we describe the proposed RRT-based planning algorithm, namely Poli-RRT*, which computes a solution to the optimal constrained planning problem, for a unicycle-like vehicle moving in a 2-dimensional Euclidean space, without linearising/approximating the nonlinear system dynamics, while obeying to actuation constraints and avoiding collisions with a-priori known static obstacles.

The following model is adopted for describing the vehicle dynamics

$$
\left\{\begin{array}{l}
\dot{x}=v \cos (\theta) \\
\dot{y}=v \sin (\theta) \\
\dot{\theta}=\omega \\
\dot{v}=a
\end{array}\right.
$$

The state $q$ of the system includes the vehicle pose $(x, y, \theta)$ and linear velocity $v$, i.e., $q=[x, y, \theta, v]^{T}$, and is confined to some bounded set $Q=\left[x_{\text {min }}, x_{\text {max }}\right] \times\left[y_{\text {min }}, y_{\text {max }}\right] \times$ $\left[\theta_{\text {min }}, \theta_{\text {max }}\right] \times\left[v_{\text {min }}, v_{\text {max }}\right]$. A value $q \in Q$ is called node according to the RRT terminology.

The control input is given by the linear acceleration $a$ and the angular velocity $\omega$, which are subject to the following constraints

$$
a \in A=\left[a_{\min }, a_{\max }\right], \omega \in \Omega=\left[\omega_{\min }, \omega_{\max }\right]
$$

Given two nodes $q$ and $q^{\prime}$, we let $e=\left(q, q^{\prime}\right)$ identify the optimal trajectory that connects $q$ to $q^{\prime}$ while minimising some cost function and satisfying the actuation constraints (2). The cost associated with $e$ is denoted by $C(e)$. According to the RRT terminology $e$ is called an edge.

System (1) is initialised at a certain node $q_{\text {start }}=$ $\left[x_{\text {start }}, y_{\text {start }}, \theta_{\text {start }}, v_{\text {start }}\right]^{T} \in Q$ and has to be steered to some goal set $Q_{\text {goal }} \subset Q$, while avoiding obstacles and following an optimal trajectory. The subset of the state space that is free of obstacles is denoted by $Q_{\text {free }}$.

Poli-RRT* first builds a tree $T=\left(Q_{T}, E_{T}\right)$, where $Q_{T} \subset$ $Q_{\text {free }}$ is a set of nodes with cardinality $N$ (defined by the user), and $E_{T}$ is a set of collision-free edges connecting nodes in $Q_{T}$. Among all sequences of nodes $q_{0}, q_{1}, q_{2}, \ldots, q_{m}$ with length $2 \leq m \leq N$, satisfying $q_{i} \in Q_{T}, i=0,1, \ldots, m, q_{0}=$ $q_{\text {start }}, q_{m} \in Q_{\text {goal }}$, and $e_{i}=\left(q_{i}, q_{i+1}\right) \in E_{T}, i=0,2, \ldots, m-1$, Poli-RRT* then chooses the one with minimal overall cost $C\left(\rightarrow q_{m}\right)=\sum_{i=0}^{m-1} C\left(e_{i}\right)$, which is a non-decreasing cost over the entire node sequence.

A key ingredient of the Poli-RRT* algorithm is represented by the procedure adopted to build the edge $e_{i}=$ $\left(q_{i}, q_{i+1}\right)$, i.e., an optimal trajectory that connects node $q_{i}$ to node $q_{i+1}$, while satisfying the actuation constraints (2), and its cost $C\left(e_{i}\right)$. This procedure rests on a two-step approach combining optimal control with a receding horizon strategy and is explained in Section III-A. The description of PoliRRT $^{*}$ algorithm is postponed to Section III-B.

\section{A. Two-step approach to edge design}

By applying to (1) the feedback linearisation strategy [21]

$$
\left[\begin{array}{l}
a \\
\omega
\end{array}\right]=\left[\begin{array}{cc}
\cos (\theta) & \sin (\theta) \\
-\frac{\sin (\theta)}{v} & \frac{\cos (\theta)}{v}
\end{array}\right]\left[\begin{array}{l}
u_{1} \\
u_{2}
\end{array}\right]
$$


we obtain the following double integrator linear model

$$
\left\{\begin{array}{l}
\dot{x}=v_{x} \\
\dot{y}=v_{y} \\
\dot{v}_{x}=u_{1} \\
\dot{v}_{y}=u_{2}
\end{array}\right.
$$

where we set $v_{x}=v \cos (\theta)$ and $v_{y}=v \sin (\theta)$. Notice that for $v=0$, the coordinate transformation presents a singularity. In turn, constraints (2) can be rewritten as

$$
\begin{aligned}
& a_{\min } \leq \cos (\theta) u_{1}+\sin (\theta) u_{2} \leq a_{\max } \\
& \omega_{\min } \leq-\frac{\sin (\theta)}{v} u_{1}+\frac{\cos (\theta)}{v} u_{2} \leq \omega_{\max }
\end{aligned}
$$

1) Optimal control without accounting for actuation constraints: Given that the feedback linearised system (4) is a double integrator with state $s=\left[x, y, v_{x}, v_{y}\right]^{T}$, we can apply the approach in [3] to obtain the minimum time trajectory connecting two states $q_{i}=\left[x_{i}, y_{i}, \theta_{i}, v_{i}\right]^{T}$ and $q_{i+1}=$ $\left[x_{i+1}, y_{i+1}, \theta_{i+1}, v_{i+1}\right]^{T}$.

The idea is to first fix the final time $\tau>0$ and determine the input $u=\left[u_{1}, u_{2}\right]^{T}:[0, \tau] \rightarrow \mathfrak{R}^{2}$ for system (4) that optimises the finite horizon cost

$$
J_{\tau}(u)=\int_{0}^{\tau}\left(1+u(t)^{T} R u(t)\right) d t
$$

with $R=R^{T} \in \mathfrak{R}^{2 \times 2}$ positive definite, subject to the constraints $s(0)=s_{i}=\left[x_{i}, y_{i}, v_{i} \cos \left(\theta_{i}\right), v_{i} \sin \left(\theta_{i}\right)\right]^{T}$ on the initial state, and $s(\tau)=s_{i+1}=$ $\left[x_{i+1}, y_{i+1}, v_{i+1} \cos \left(\theta_{i+1}\right), v_{i+1} \sin \left(\theta_{i+1}\right)\right]^{T}$ on the final state. Let $J_{\tau}^{\star}$ be the resulting optimal cost, i.e.,

$$
J_{\tau}^{\star}=\min _{u:[0, \tau] \rightarrow \Re^{2}} \int_{0}^{\tau}\left(1+u(t)^{T} R u(t)\right) d t .
$$

Then, the optimal value $\tau^{\star}$ for $\tau$ can be obtained by minimising $J_{\tau}^{\star}$. Correspondingly, one can obtain the optimal input $u^{\star}$ and state $s^{\star}=\left[x^{\star}, y^{\star}, v_{x}^{\star}, v_{y}^{\star}\right]^{T}$, which, in turn, maps back to the optimal $q^{\star}=\left[x^{\star}, y^{\star}, \theta^{\star}, v^{\star}\right]^{T}$. In particular, from [3], we have the following analytic expression for $J_{\tau}^{\star}$ :

$$
J_{\tau}^{\star}=\tau+\left(s_{i+1}-\exp (A t) s_{i}\right)^{T} G(\tau)\left(s_{i+1}-\exp (A t) s_{i}\right),
$$

where $G(\tau)$ is the weighted controllability Gramian

$$
G(\tau)=\int_{0}^{\tau} \exp (A(\tau-t)) B R^{-1} B^{T} \exp \left(A^{T}(\tau-t)\right) d t .
$$

Once $\tau^{\star}=\operatorname{argmin}_{\tau>0} J_{\tau}^{\star}$ is determined, then, $u^{\star}(t)$ and $s^{\star}(t)$, $t \in\left[0, \tau^{\star}\right]$, are given by:

$$
\begin{aligned}
& u^{\star}(t)=R^{-1} B^{T} \exp \left(A^{T}\left(\tau^{\star}-t\right)\right) d^{\star} \\
& s^{\star}(t)=\left[\begin{array}{ll}
I_{4} & 0_{4}
\end{array}\right] \exp \left(\left[\begin{array}{cc}
A & B R^{-1} B^{T} \\
0_{4} & -A^{T}
\end{array}\right]\left(t-\tau^{\star}\right)\right)\left[\begin{array}{c}
s_{i+1} \\
d^{\star}
\end{array}\right]
\end{aligned}
$$

where $I_{4}$ is the $4 \times 4$ identity matrix and $0_{4}$ is the $4 \times 4$ zero matrix and we set $d^{\star}=G\left(\tau^{\star}\right)^{-1}\left(s_{i+1}-\exp \left(A \tau^{\star}\right) s_{i}\right)$.

Let $q^{\star}(t), t \in\left[0, \tau^{\star}\right]$, be the optimal trajectory $s^{\star}(t), t \in$ $\left[0, \tau^{\star}\right]$, mapped back to the original state coordinates of the unicycle model (1). If the actuation constraints (5) are satisfied by $u^{\star}(t)$ and $q^{\star}(t)$, for all $t \in\left[0, \tau^{\star}\right]$, then, the edge $e_{i}=\left(q_{i}, q_{i+1}\right)$ is given by the optimal trajectory $q^{\star}$ that takes the system from node $q_{i}$ to node $q_{i+1}$ within a time interval of length $\tau^{\star}$ and a cost $C\left(e_{i}\right)=J_{\tau^{\star}}^{\star}=J_{\tau^{\star}}\left(u^{\star}\right)$. If that is not the case, a receding horizon strategy is put in place so as to force the constraints (5) to hold, while keeping the resulting trajectory close to the optimal one along a time horizon of length $\tau^{\star}$. This is explained in the following.

2) Receding horizon with actuation constraints: To simplify computation, we introduce a discrete time version of the feedback linearised system (4):

$$
\left\{\begin{array}{l}
x(k+1)=x(k)+v_{x}(k) \Delta \\
y(k+1)=y(k)+v_{y}(k) \Delta \\
v_{x}(k+1)=v_{x}(k)+u_{1}(k) \Delta \\
v_{y}(k+1)=v_{y}(k)+u_{2}(k) \Delta
\end{array}\right.
$$

where $\Delta>0$ is the sample time interval. By setting $s(k)=$ $\left[x(k), y(k), v_{x}(k), v_{y}(k)\right]^{T}$ and $u(k)=\left[u_{1}(k), u_{2}(k)\right]^{T}$, system (8) can be rewritten in the compact form

$$
s(k+1)=F s(k)+H u(k)
$$

where $F$ and $H$ are appropriately defined matrices.

Let $k_{f}:=\left\lfloor\frac{\tau^{\star}}{\Delta}\right\rfloor$ be the discrete time version of the final time $\tau^{\star}$ as computed in Section III-A.1.

Our goal is to choose $u(0), u(1), \ldots, u\left(k_{f}-1\right)$ so as to keep $s(k)$ close to the optimal constrained-free trajectory $s^{\star}(k \Delta)$ computed in Section III-A.1, while forcing the constraints (5) to hold at each discrete time instant $k$ along the discrete time horizon $\left[0, k_{f}\right]$.

To this purpose, we introduce the following constrained optimisation problem $C-O P T(k)$ :

$$
\min _{u(k), u(k+1), \ldots, u\left(k_{f}-1\right)} \sum_{h=k+1}^{k_{f}}\left(s(h)-\bar{s}_{h}\right)^{T} W_{h}\left(s(h)-\bar{s}_{h}\right)
$$

subject to:

$$
\begin{aligned}
& s(h+1)=F s(h)+H u(h) \\
& s(k)=\bar{s}_{k} \\
& a_{\text {min }} \leq\left[\cos \left(\bar{\theta}_{h}\right) \sin \left(\bar{\theta}_{h}\right)\right] u(h) \leq a_{\text {max }} \\
& \omega_{\text {min }} \leq\left[-\frac{\sin \left(\bar{\theta}_{h}\right)}{\bar{v}_{h}} \frac{\cos \left(\bar{\theta}_{h}\right)}{\bar{v}_{h}}\right] u(h) \leq \omega_{\max } \\
& \forall h=k, \ldots, k_{f}-1,
\end{aligned}
$$

with $W_{h}, h=k+1, k+2, \ldots, k_{f}$, positive definite matrices of dimension 4. Once $\bar{s}_{h}, h=k, k+1, \ldots k_{f}$, and $\bar{\theta}_{h}$ and $\bar{v}_{h}$, $h=k, k+1, \ldots k_{f}-1$, are given, $C-O P T(k)$ is an easy to solve convex optimisation problem with quadratic cost $(s(h)$ is a linear function of the optimisation variables) and linear constraints.

Let $u^{\star, k}(h), h=k, \ldots k_{f}-1$, be the solution $C-O P T(k)$, and $s^{\star, k}(h), h=k, \ldots k_{f}$, the trajectory obtained by applying $u^{\star, k}$ to (8), starting from $\bar{s}_{k}$ at time $k$.

The receding horizon strategy then unfolds as follows. We initially solve the $C-O P T(k)$ for $k=0$ by setting

$$
\begin{aligned}
& \bar{s}_{h}=s^{\star}(h \Delta), h=0, \ldots, k_{f} \\
& \bar{\theta}_{h}=\theta^{\star}(h \Delta), h=0, \ldots, k_{f}-1 \\
& \bar{v}_{h}=v^{\star}(h \Delta), h=0, \ldots, k_{f}-1
\end{aligned}
$$


where $s^{\star}$ (and, correspondingly, $\theta^{\star}$ and $v^{\star}$ ) is the optimal constrained-free trajectory computed in Section III-A.1. The obtained solution $u^{\star, k}, k=0$, will necessarily satisfy the constraints (5) at time $k$ since the trajectory $s^{\star, k}$ satisfies $s^{\star, k}(k)=$ $\bar{s}_{k}$ and constraints (5) are imposed in the $C-O P T(k)$ by using $\bar{\theta}_{h}$ and $\bar{v}_{h}, h=k, k+1, \ldots k_{f}-1$, derived from $\bar{s}_{h}, h=$ $k, k+1, \ldots k_{f}$. However, the solution $u^{\star, k}$ will not necessarily satisfy the constraints at time $k+1, k+2, \ldots, k_{f}-1$. This is because, in general $s^{\star, k}(h)$ will be different from $\bar{s}_{h}$ for $h=k+1, k+2, \ldots, k_{f}-1$.

If $u^{\star, k}, k=0$, does satisfy the constraints at time $k+$ $1, k+2, \ldots, k_{f}-1$, then, we have a feasible solution and we can define the edge $e_{i}$ as given by the optimal constrained trajectory $q^{\star, k}$ that takes the system from node $q_{i}$ to a node close to $q_{i+1}$ (the larger is the weighting matrix $W_{k_{f}}$ the closer the system will get to $q_{i+1}$ ) within a time interval of length $\tau^{\star}$ with a cost $C\left(e_{i}\right)=J_{k_{f} \Delta}\left(u^{\star, k}\right)$ obtained by plugging into (6) a piece-wise constant version of $u^{\star, k}$.

If, instead, $u^{\star, k}, k=0$, does not satisfy the constraints at some time $h \in\left[k+1, k+2, \ldots, k_{f}-1\right]$, then, we do not have a feasible solution yet. We hence apply only the first input sample $u^{\star, k}(k)$, and determine the further input values by solving the $C-O P T(k)$ for $k=1$ with

$$
\begin{aligned}
& \bar{s}_{h}=s^{\star, k-1}(h \Delta), h=k, \ldots, k_{f} \\
& \bar{\theta}_{h}=\theta^{\star, k-1}(h \Delta), h=k, \ldots, k_{f}-1 \\
& \bar{v}_{h}=v^{\star, k-1}(h \Delta), h=k, \ldots, k_{f}-1 .
\end{aligned}
$$

Again if $u^{\star, k}, k=1$, does satisfy the constraints at all times $k+1, k+2, \ldots, k_{f}-1$, then, we have a feasible solution and we can define the edge $e_{i}$ as the optimal constrained trajectory $q^{\star, k}$ that takes the system from node $q_{i}$ to a node close to $q_{i+1}$ through $u(0)=u^{\star, 0}(0), u(h)=u^{\star, 1}(h)$, $h=1,2, \ldots, k_{f}-1$. The cost associated to $e_{i}$ will be given by $C\left(e_{i}\right)=J_{k_{f} \Delta}\left(\left[u^{\star, 0}(0), u^{\star, 1}(1), \ldots, u^{\star, 1}\left(k_{f}-1\right)\right]^{T}\right)$.

If, instead, $u^{\star, k}, k=1$, does satisfy the constraints at some time $h \in\left[k+1, k+2, \ldots, k_{f}-1\right]$, then, we apply only the input sample $u^{\star, k}(k)$, increment $k$ to $k=2$, and determine the further input values by solving the $C-O P T(k)$ with $\bar{s}_{h}, h=$ $k, k+1, \ldots k_{f}$, and $\bar{\theta}_{h}$ and $\bar{v}_{h}, h=k, k+1, \ldots k_{f}-1$, defined in (10).

This kind of reasoning is repeated until a feasible solution is found at some iteration $k<k_{f}-1$ or $k=k_{f}-1$ is reached.

\section{B. Poli-RRT* Algorithm}

The Poli-RRT* algorithm works as follows:

1) Tree initialisation: an empty tree $T=\left(Q_{T}, E_{T}\right)$ is instantiated, where $Q_{T}$ is the set of nodes and $E_{T}$ is the set of edges connecting the nodes. Then, node $q_{0}=q_{\text {start }}$ is added to $T: Q_{T}=\left\{q_{\text {start }}\right\}$;

2) Random sampling: a state configuration $q_{\text {rand }}$ is randomly sampled within the free portion of the state space $Q_{\text {free }}$ according to an uniform distribution;

3) Neighbour radius computation: the neighbour radius $r$, used to limit the search for optimal edges in steps 4) and 5) of the algorithm, is determined as:

$$
r=\underset{q \in Q_{\text {reach }}}{\operatorname{argmax}}\left(\max \left\{C\left(e_{q, 1}\right), C\left(e_{q, 2}\right)\right\}\right),
$$

where $e_{q, 1}=\left(q, q_{\text {rand }}\right), e_{q, 2}=\left(q_{\text {rand }}, q\right)$ and $Q_{\text {reach }}$ is defined as the subset of $Q_{T}$ containing all the nodes that are inside a $d$-dimensional ball of radius $\gamma_{\text {ball }}$, centered in $q_{\text {rand }}$ :

$$
Q_{\text {reach }}=\left\{q \in Q_{T} \mid\left\|q_{\text {rand }}-q\right\|_{2} \leq \gamma_{\text {ball }}\right\} .
$$

Constant $\gamma_{\text {ball }}$ is given by $\gamma_{\text {ball }}=\gamma_{R R T^{*}}\left(\log \left|Q_{T}\right| /\left|Q_{T}\right|\right)^{1 / d}$, where $d=4$ is the state space dimension and $\gamma_{R R T^{*}}>2(1+1 / d)^{1 / d}\left(\mu\left(Q_{\text {free }}\right) / \eta_{d}\right)^{1 / d}$, with $\mu\left(Q_{\text {free }}\right)$ being the volume of $Q_{\text {free }}$ and $\eta_{d}$ being the volume the unit ball in the $d$-dimensional Euclidean space, respectively (see [1], [12] for further details).

4) Minimum-cost path selection: in order to connect $q_{\text {rand }}$ to the tree along a minimum-cost path the following steps are taken:

- the minimum cost collision-free edge $e_{\min }=$ $\left(q_{\text {min }}, q_{\text {rand }}\right)$ among the edges $e=\left(q, q_{\text {rand }}\right), q \in Q_{T}$, is determined by computing

$$
q_{\text {min }}=\underset{q \in\left\{q \in Q_{T} \mid C(e) \leq r \wedge \text { Collisionfree }(e)\right\}}{\operatorname{argmin}}(C(\rightarrow q)+C(e)),
$$

where $r$ is the neighbour radius, CollisionFree $(e)$ is a function that returns true when $e$ is a collision-free trajectory, false otherwise, and $C(\rightarrow q)$ represents the total cost of the current-best path going from $q_{\text {start }}$ to $q$

- $q_{\text {rand }}$ and $e_{\text {min }}$ are added to $T$ :

$$
Q_{T}=Q_{T} \cup\left\{q_{\text {rand }}\right\}, \quad E_{T}=E_{T} \cup\left\{e_{\text {min }}\right\}
$$

5) Tree rewiring: every time a new node $q_{\text {rand }}$ is added to the tree it is necessary to check if there exist a minimumcost path reaching any other node inside the tree and passing through $q_{\text {rand }}$. More in detail, for every node $q \in Q_{T}$, if $e=\left(q_{\text {rand }}, q\right)$ satisfies CollisionFree $(e)=$ true, $C(e) \leq r$, and $C\left(\rightarrow q_{\text {rand }}\right)+C(e)<C(\rightarrow q)$, then, the tree is rewired:

$$
E_{T}=\left\{E_{T} \backslash\left\{e_{\text {prev }}\right\}\right\} \cup\{e\},
$$

where $e_{\text {prev }}=(\operatorname{Parent}(q), q)$ is the previous edge connecting $q$ to the tree and Parent $(q)$ is a function returning the node from which edge $e_{\text {prev }}$ starts.

6) Termination: the algorithm keeps iterating steps 2), 3), 4) and 5) until $\left|Q_{T}\right|=N$ and then it stops;

7) Optimal trajectory: if the goal area has been reached, the minimum cost-to-go node inside $Q_{\text {goal }}$ is selected:

$$
Q_{\text {goal }} \cap Q_{T} \neq \emptyset \Longrightarrow q_{\text {goal }}=\underset{q \in\left(Q_{\text {goal }} \cap Q_{T}\right)}{\operatorname{argmin}} C(\rightarrow q)
$$

and the trajectory $e_{\text {goal }}=\left(q_{\text {start }}, q_{\text {goal }}\right)$ connecting $q_{\text {start }}$ with $q_{\text {goal }}$ is returned along with the entire tree $T$.

Remark Note that at steps 4 and 5 in Poli-RRT* algorithm, condition $C(e)<r$ needs to be verified on an edge $e=\left(q, q^{\prime}\right)$ involving some node that is candidate for further processing. Violation of such a condition is easy to evaluate since it holds that $C(e) \geq J_{\tau^{\star}}^{\star}=\min _{\tau \geq 0} J_{\tau}^{\star}$, where $J_{\tau}^{\star}$ is the cost of an 
unconstrained optimal trajectory driving system (1) from $q$ to $q^{\prime}$ in an interval of length $\tau$ and is given in analytic form in (7). Thus, the adoption of the neighbour radius $r$ introduced at step 3 is effective in reducing the number of nodes to be processed at each iteration of the algorithm. Furthermore, it does not modify the outcome of the algorithm in terms of the computed optimal trajectory [1], [12].

\section{Simulation Results}

A MATLAB ${ }^{\odot}$ implementation of the proposed algorithm was developed and tested, considering the dynamic model (1), with the state bounded as follows: $x \in[0,100], y \in$ $[0,100], \theta \in[-\pi,+\pi]$, and $v \in[\varepsilon, 1]$, where $\varepsilon>0$ ensures that linearisation (3) is always well-defined. In order to solve the constraint-free optimal control problem (see Section IIIA.1), a matrix $R=10 I_{2}$ equally penalising linear acceleration $a$ and angular velocity $\omega$ was chosen. As for the receding horizon strategy in Section III-A.2, eventually refining the solution to the constraint-free optimal control problem so as to impose the saturation constraints, a sample time interval $\Delta=0.1 s$ was selected. Furthermore, matrices $W_{h}$, $h=1, \ldots, k_{f}$, in the $C-O P T(k)$ problem (9) were set to $W_{h}=10 I_{4}, \quad h=1, \ldots, k_{f}-1$, and $W_{k_{f}}=100 I_{4}$, so as to assign a stronger weight to the tracking error at the last time instant $k_{f}$ with respect to that at the previous time instants $h=1, \ldots, k_{f}-1$. Finally, given the dimension of the state space and the upper and lower bounds on state variables, we imposed $\gamma_{R R T^{*}}=24.71$.

The virtual environment used as a test-bed is depicted in Figure 2(a), and it is composed of a start configuration, a goal area, and four non-overlapping circular obstacles.

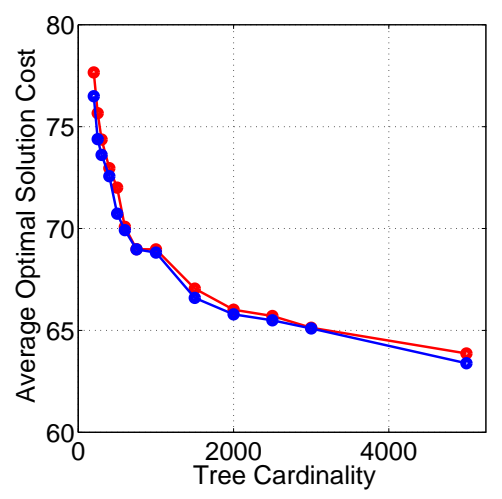

Fig. 1. Average cost of optimal solution as a function of tree cardinality with actuation limits $A_{1}, \Omega_{1}$ (blue line), and $A_{2}, \Omega_{2}$ (red line).

The algorithm has been tested considering different values of the maximum tree cardinality $N$, i.e., 200, 250, 300, 400, 500, 600, 750, 1000, 1500, 2000, 2500, 3000 and 5000 nodes. For each of these values, several runs were realised imposing two different actuation constraints (see equation (2)): the former characterised by larger bounds, $A_{1}=[-0.50,0.50]$ and $\Omega_{1}=[-0.50,0.50]$; the latter by more strict limits, $A_{2}=[-0.20,0.20]$ and $\Omega_{2}=[-0.20,0.20]$.
Figure 2 shows the trees obtained considering different maximum cardinality values and imposing actuation constraints $A_{1}$ and $\Omega_{1}$.

Figures 3(a) and 3(b) show the actuation profiles (linear acceleration $a$ and angular velocity $\omega$ ) corresponding to the optimal trajectory found with maximum tree cardinality $N$ set to 1000 nodes and imposing the actuation limits $A_{1}$ and $\Omega_{1}$. The pictures clearly show that the values of the control variables never exceed the upper and lower bounds. On the other hand, Figures 3(c) and 3(d) show the actuation profiles corresponding to a different optimal trajectory obtained considering the same maximum tree cardinality, but $A_{2}$ and $\Omega_{2}$ as actuation limits. In this case, the actuation profiles partially saturate the upper/lower bounds, but they still never exceed them.

Finally, Figure 1 shows the average cost of the optimal solution over multiple runs performed with the same maximum cardinality. As expected, not only the average cost decreases with respect to the maximum tree cardinality, but a better average cost is achieved while considering as actuation limits $A_{1}$ and $\Omega_{1}$, rather than the more restrictive $A_{2}$ and $\Omega_{2}$.

\section{CONCLUSIONS}

This paper proposes the first RRT-based planner able to compute an optimal and dynamically feasible trajectory, considering differential and actuation constraints, without representing the system dynamics with an approximate linearised model, but relying on an exact linearisation. This approach was used to generate feasible and optimal trajectories for a unicycle-like vehicle moving in a virtual environment populated by obstacles.

An extension to hybrid dynamic models and an efficient implementation of the planner, adopting an heuristic that enables a branch-and-bound approach so as to avoid attempts to connect nodes that cannot contribute to an optimal solution, will be considered as future developments.

\section{REFERENCES}

[1] S. Karaman and E. Frazzoli, "Optimal kinodynamic motion planning using incremental sampling-based methods," in IEEE Conference on Decision and Control (CDC), Atlanta, GA, 2010.

[2] J. H. Jeon, S. Karaman, and E. Frazzoli, "Anytime computation of time-optimal off-road vehicle maneuvers using the RRT*," in IEEE Conference on Decision and Control, 2011, pp. 3276-3282.

[3] D. J. Webb and J. V. D. Berg, "Kinodynamic RRT*: Optimal motion planning for systems with linear differential constraints," CoRR, vol. abs/1205.5088, 2012.

[4] A. Perez, R. Platt, G. Konidaris, L. Kaelbling, and T. Lozano-Perez, "LQR-RRT*: Optimal sampling-based motion planning with automatically derived extension heuristics," in IEEE International Conference on Robotics and Automation, 2012, pp. 2537-2542.

[5] G. Goretkin, A. Perez, R. Platt, and G. Konidaris, "Optimal samplingbased planning for linear-quadratic kinodynamic systems," in IEEE Conference on Robotics and Automation, 2013.

[6] R. Burden and D. Faires, Numerical Analysis. Brooks/Cole, 2001.

[7] M. Pivtoraiko, R. Knepper, and A. Kelly, "Differentially constrained mobile robot motion planning in state lattices," Journal of Field Robotics, vol. 26, pp. 308-333, 2009.

[8] M. Likhachev and D. Ferguson, "Planning long dynamically feasible maneuvers for autonomous vehicles," The International Journal of Robotic Research, vol. 28, pp. 933-945, 2009.

[9] L. Kavraki, P. Svestka, J.-C. Latombe, and M. Overmars, "Probabilistic roadmaps for path planning in high-dimensional configuration spaces," IEEE Tran. Robot. Autom., vol. 12, no. 4, pp. 566-580, 1996. 


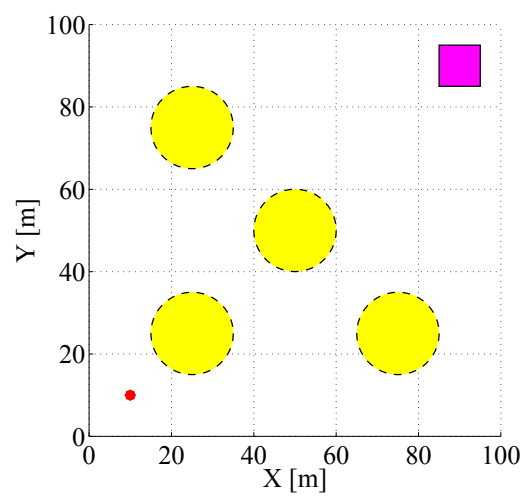

(a) Virtual environment

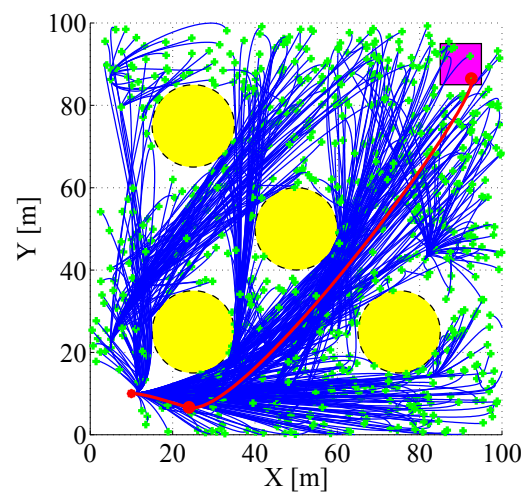

(d) 750 nodes

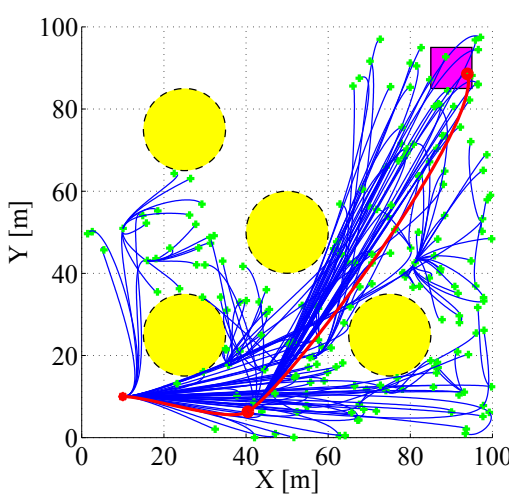

(b) 200 nodes

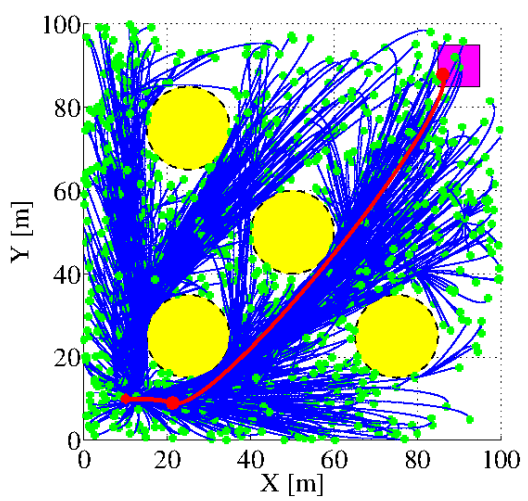

(e) 1000 nodes

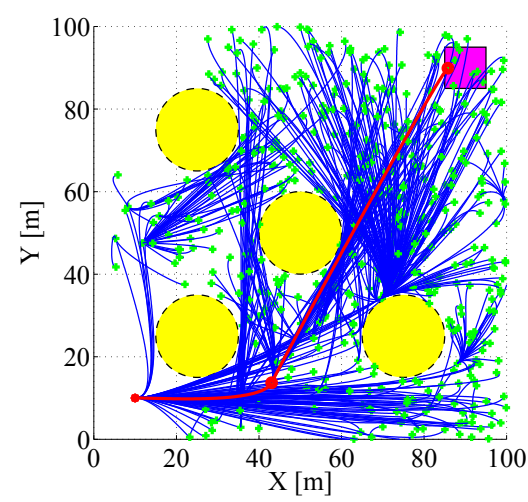

(c) 500 nodes

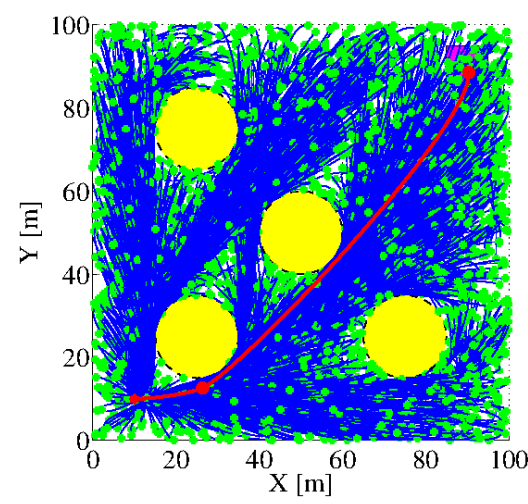

(f) 3000 nodes

Fig. 2. A planning experiment: start pose (red dot), goal area (magenta square), obstacles (yellow circles), tree nodes (green crosses), tree edges (solid blue), optimal solution (solid red), optimal trajectory nodes (red circles).

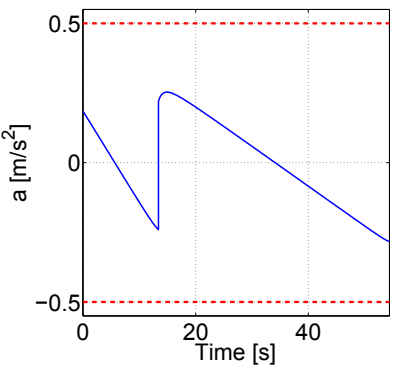

(a) Acceleration limits $A_{1}$

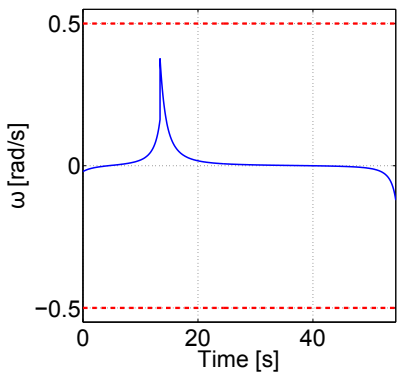

(b) Angular velocity limits $\Omega_{1}$

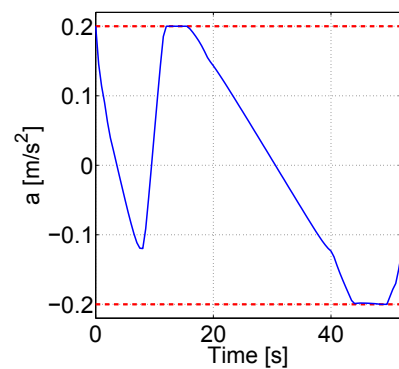

(c) Acceleration limits $A_{2}$

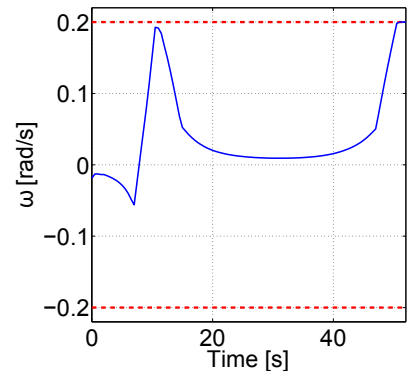

(d) Angular velocity limits $\Omega_{2}$

Fig. 3. Actuation profiles of the optimal solutions obtained with $N=1000$ (solid blue) and corresponding actuation limits (dashed red).

[10] E. Kavraki, M. Kolountzakis, and J.-C. Latombe, "Analysis of probabilistic roadmaps for path planning," IEEE Transactions on Robotics and Automation, vol. 14, no. 1, pp. 166-171, 1998.

[11] S. M. LaValle and J. J. Kuffner, "Randomized kinodynamic planning," Int. J. Robot. Res., vol. 20, no. 5, pp. 378-400, 2001.

[12] S. Karaman and E. Frazzoli, "Incremental sampling-based algorithms for optimal motion planning," in Robotics: Science and Systems, 2010.

[13] S. Karaman, M. Walter, A. Perez, E. Frazzoli, and S. Teller, "Real-time motion planning using the RRT*," in IEEE Conference on Robotics and Automation, 2011.

[14] S. Karaman and E. Frazzoli, "Sampling-based algorithms for optimal motion planning," Int. J. Rob. Res., vol. 30, no. 7, pp. 846-894, 2011.

[15] A. Perez, S. Karaman, M. Walter, A. Shkolnik, E. Frazzoli, and S. Teller, "Asymptotically-optimal path planning for manipulation using incremental sampling-based algorithms," in IEEE/RSJ International Conference on Intelligent Robots and Systems, 2011.

[16] S. Karaman and E. Frazzoli, "Sampling-based optimal motion planning with deterministic $\mu$-calculus specifications," in American Control
Conference, 2012.

[17] Frazzoli, E., Dahleh, M.A, and E. Feron, "Real-time motion planning for agile autonomous vehicles," in American Control Conference, vol. 1, 2001, pp. 43-49.

[18] M. Branicky, M. Curtiss, J. Levine, and S. Morgan, "RRTs for nonlinear, discrete, and hybrid planning and control," in IEEE Conference on Decision and Control, vol. 1, 2003, pp. 657-663.

[19] — - "Sampling-based planning, control and verification of hybrid systems," IEE Proceedings Control Theory and Applications, vol. 153 no. 5, pp. 575-590, 2006.

[20] Y. Abbasi-Yadkori, J. Modayil, and C. Szepesvari, "Extending rapidlyexploring random trees for asymptotically optimal anytime motion planning," in IEEE/RSJ Int. Conf. Int. Rob. Sys., 2010, pp. 127-132.

[21] D. Stipanovic, G. Inalhan, R. Teo, and C. Tomlin, "Decentralized overlapping control of a formation of unmanned aerial vehicles," Automatica, vol. 40, no. 8, pp. 1285-1296, 2004. 